Conclusion These results indicate that in principle $\mathrm{VO}_{2 \max }$ in $\mathrm{ml} / \mathrm{kg} / \mathrm{min}$ as an indirect indicator of cardiac function or for cardiac transplantation selection is unreliable when applied to overweight heart failure patients. Extending this concept to the entire spectrum of body weights, the practice of correcting $\mathrm{Vo}_{2 \max }$ by body weight in cardiological practice would also require urgent reconsideration.

\section{PRESSURE VS FLOW AS A GUIDE FOR PACEMAKER OPTIMISATION? THE ACUTE HAEMIODYNAMIC EFFECTS OF CHANGES TO ATRIOVENTRICULAR DELAY}

doi:10.1136/heartjn|-2011-300198.100

C H Manisty, B Unsworth, R Baruah, P Pabari, Z I Whinnett, J Mayet, D P Francis. Imperial College, St. Marys Hospital, London, UK

Background Non-invasive blood pressure monitoring by continuous finger photoplethysmography (Finometer) may have value in pacemaker

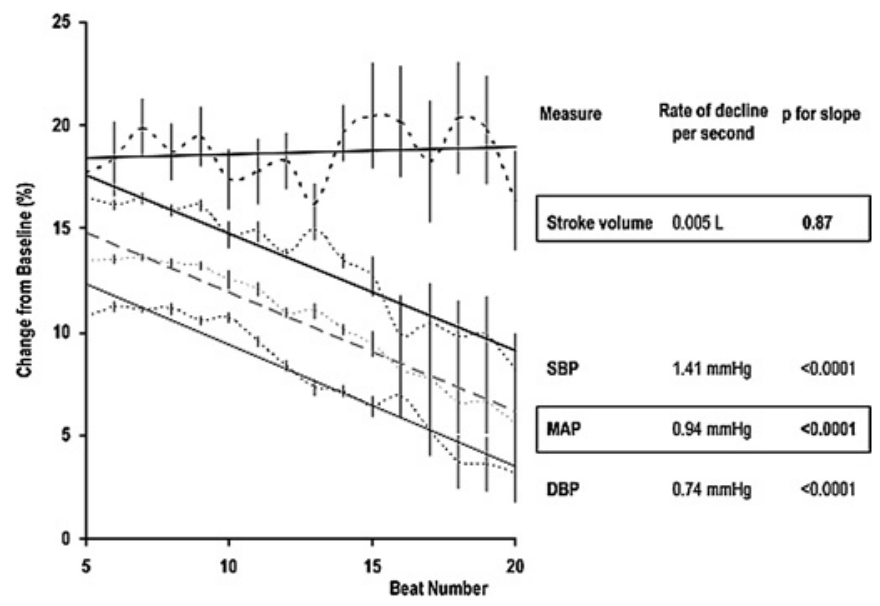

Abstract 100 Figure 1

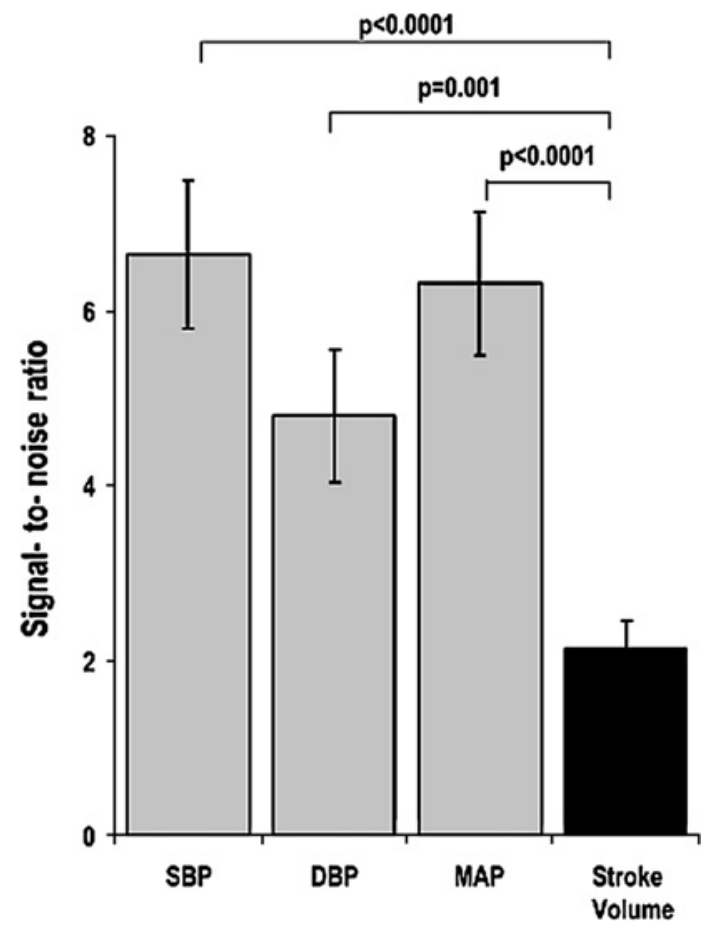

Abstract 100 Figure 2 optimisation. However, the immediate increment in blood pressure seems to diminish somewhat in the initial minute: it is unclear whether this is due to an (undesirable) fall in stroke volume or a (desirable) compensatory reduction in peripheral resistance.

Methods and Results We studied this question by measuring beat-bybeat stroke volume (flow) using Doppler echocardiography, and blood pressure using continuous finger photoplethysmography, during and after atrioventricular delay adjustment from 40 to $120 \mathrm{~ms}$ in 19 subjects with cardiac pacemakers. Quintuplicate experimental runs were performed. Blood pressure and stroke volume (flow) both increased immediately $(\mathrm{p}<0.00001$ within one heartbeat). The immediate pressure increment correlated strongly with the immediate flow increment $(r=0.74, p=0.0001)$. Pressure showed a partial decline a few seconds later (average rate $0.65 \mathrm{~mm}$ $\mathrm{Hg} /$ beat, $\mathrm{r}=-0.98, \mathrm{p}<0.0001$ ), in contrast, flow did not decline $(\mathrm{p}=\mathrm{NS})$, Abstract 100 figure 1. Signal-to-noise ratio was significantly better for pressure than flow $(6.3 \pm 3.6$ vs $2.1 \pm 1.4, \mathrm{p}<0.0001)$, Abstract 100 figure 2

Conclusions Improving atrioventricular delay immediately increases blood pressure; however this effect decays slightly over the subsequent minute. This is due to compensatory vasodilatation rather than a reduction in cardiac function. Pressure changes are simpler to measure and easier to distinguish from random variation than Doppler measurements of flow, but are best measured immediately, before the vascular compensation.

\section{WHAT DEGREE OF PULMONARY HYPERTENSION PREDICTS POOR OUTCOME IN PATIENTS WITH LEFT VENTRICULAR SYSTOLIC DYSFUNCTION? A 10-YEAR FOLLOW-UP STUDY}

doi:10.1136/heartjnl-2011-300198.101

${ }^{1} \mathrm{~B}$ R Szwejkowski, ${ }^{1} \mathrm{D}$ H J Elder, ${ }^{1} \mathrm{~A}$ M J Choy, ${ }^{2}$ S D Pringle, ${ }^{1} \mathrm{~A}$ D Struthers, ${ }^{1} \mathrm{C}$ C Lang. ${ }^{1}$ University of Dundee, Dundee, UK; ${ }^{2}$ Department of Cardiology, NHS Tayside, Dundee

Introduction The presence of pulmonary hypertension in left ventricular systolic dysfunction (LVSD) is an ominous sign. It remains unclear the level at which pulmonary hypertension conveys a mortality risk in patients with LVSD.

Methods We performed a record-linkage study in Tayside, UK (population approximately 400000 ) utilising the Tayside echocardiogram database ( $>100000$ echo's) maintained by the Health Informatics Centre (HIC). Datasets from HIC include mortality data and other health care activities linked anonymously by the community health index (CHI) number. Patients were included in the analysis if they had LVSD and had a right ventricular systolic pressure (RVSP) measurement. Cox proportional hazards regression analysis was used to examine the effects of different ranges of RVSP measures on all cause mortality.

Results 2910 patients (mean age, $74.5 \pm 11.4$ years; $43 \%$ male) met entry criteria. Mean RVSP was $43.3 \pm 12.7 \mathrm{~mm} \mathrm{Hg}$ and median follow was 362 days (IOR 129-850 days). There was a significant correlation between RVSP and survival ( $p<0.0001)$. In quartiles of RVSP, the HR after adjustment for confounding factors including LVSD and the presence of chronic obstructive pulmonary disease (COPD) were: RVSP 35-41 mm Hg, HR 1.12 (95\% CI 0.95 to 1.32, $\mathrm{p}=0.175$ ), RVSP 42-50 mm Hg, 1.27 (1.07 to 1.49, $\mathrm{p}<0.001$ ) and RVSP 51-106 mm Hg 1.62 (1.38 to 1.1, p<0.001). For each $5 \mathrm{~mm}$ $\mathrm{Hg}$ stepwise increase in RVSP the HR for all cause mortality was 1.07 (1.04 to $1.09, \mathrm{p}<0.001$ ). Abstract 101 figure 1 shows the Kaplan-Meier survival curves for all cause mortality for all patients expressed as different RVSP quartiles. 


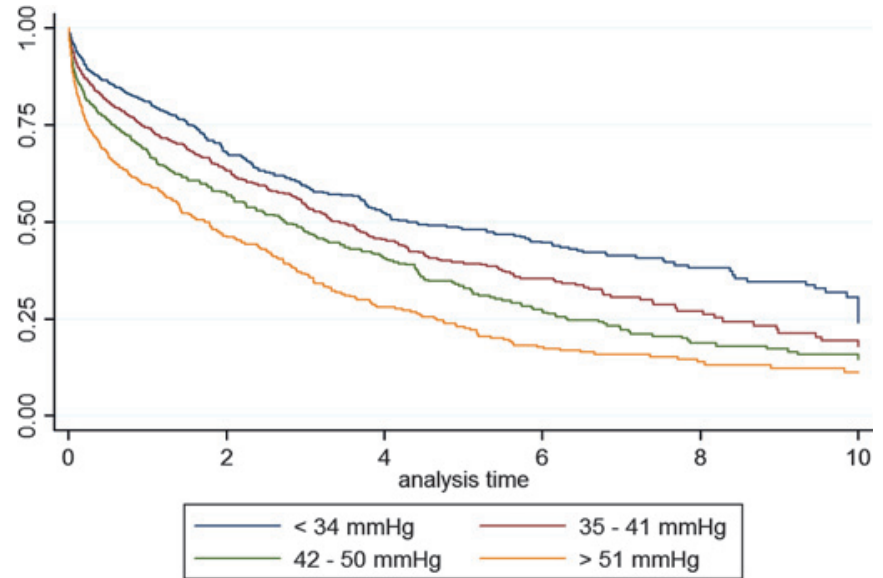

Abstract 101 Figure 1 Survival of RVSP quartile.

Conclusion An RVSP of greater than $42 \mathrm{~mm} \mathrm{Hg}$ is predictive of increased mortality in heart failure. This is finding is independent of LVSD and COPD.

\section{ETHNIC DIFFERENCES IN ENDOTHELIAL FUNCTION IN CHRONIC HEART FAILURE}

doi:10.1136/heartjnl-2011-300198.102

${ }^{1} \mathrm{E}$ Shantsila, ${ }^{2} \mathrm{P}$ S Gill, ${ }^{3} \mathrm{G}$ Y H Lip. ${ }^{1}$ University of Birmingham Centre for Cardiovascular Sciences, City Hospital, Birmingham, UK; ${ }^{2}$ University of Birmingham, Primary Care and Populational Sciences, Birmingham, UK; ${ }^{3}$ University of Birmingham Centre for Cardiovascular Science, Birmingham, UK

Background Endothelial dysfunction is characteristic of patients with heart failure (HF) and is associated with an increased risk of future cardiovascular events. However, data on ethnic differences in endothelial function in HF are scarce. In this study we aimed to compare parameters of macro- and micro-vascular endothelial function and arterial elasticity in HF age- and sex-matched patients of different ethnic origin: (i) white European, (ii) south Asian and (iii) African-Caribbean. Additionally, SA patients with systolic HF were compared to two matched control groups: (i) south Asian patients with coronary artery disease without HF(disease controls) and (ii) south Asian "healthy controls".

Methods We recruited 186 age/sex-matched patients with HF (ejection fraction $<40 \%$ ) of SA ( $n=43$, age $66.5 \pm 11.1$ years), white $(n=44$, age $68.4 \pm 9.4$ years) and African-Caribbean ( $n=21$, age 69.2 \pm 10.3 years)

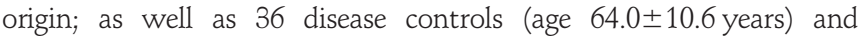
40 healthy controls ( $n=40$, age $63.3 \pm 9.24$ years). Macrovascular endothelial function was assessed as brachial artery flow mediated dilation in response to hyperaemia (FMD) and glyceryltrinitrate were assessed by vascular ultrasonography (iE33, Philips, USA). Microvascular endothelial function was evaluated by laser Doppler flowmetry of forearm skin (DRT4, Moor Instruments, UK) after iontophoresis of acetylcholine and sodium nitroprusside. Arterial stiffness was quantified by pulse wave velocity and augmentation index using (Sphygmocor, Australia).

Results Compared to disease controls and healthy controls south Asian patients with HF had impaired microvascular response to acetylcholine $(390 \pm 302 \%, 549 \pm 264 \%$, and $123 \pm 95.5 \%$, respectively, $\mathrm{p}<0.05)$ and reduced FMD $(7.12 \pm 3.64 \%, 11.8 \pm 4.66 \%$, and $4.86 \pm 4.88 \%$, respectively). HF patients of south Asian origin had impaired microvascular endothelial function (response to acetylcholine123 $\pm 95.5 \%$ ) compared to white $(258 \pm 15.6 \%)$ and African-Caribbean $(286 \pm 17.3 \%)$ groups ( $p>0.05)$. HF patients of white origin had higher FMD than south Asian (4.86 $\pm 4.88 \%)$ and African-Caribbean $(5.36 \pm 3.24 \%)$ patients $(p<0.05)$. No difference in glyceryltrinitrate- and sodium nitroprusside-mediated (endothelialindependent) response was observed between study groups. In south Asian subjects, parameters of pulse wave velocity and augmentation index did not differ between those with HF and those in control groups. No ethnic differences were detected in pulse wave velocity. Conclusion: South Asian patients with HF have impaired micro- and macro-vascular endothelial function, but preserved arterial elastic properties. Significant ethnic differences in endothelial function are present in patients with HF.

\section{SENILE SYSTEMIC AMYLOIDOSIS: A COMMON CAUSE OF HEART FAILURE IN THE ELDERLY?}

doi:10.1136/heartjnl-2011-300198.103

${ }^{1} \mathrm{~J} \mathrm{H}$ Pinney, ${ }^{2} \mathrm{H} \mathrm{J}$ Lachmann, ${ }^{2} \mathrm{~J} \mathrm{D}$ Gillmore, ${ }^{2} \mathrm{~A}$ Wechalekar, ${ }^{3} \mathrm{~S} \mathrm{D} \mathrm{J}$ Gibbs, ${ }^{3} \mathrm{P}$ Sattianayagam, ${ }^{4,5} \mathrm{~S} \mathrm{M}$ Banypersad, ${ }^{6,7} \mathrm{~J}$ Dungu, ${ }^{3} \mathrm{~N}$ Wassef, ${ }^{3} \mathrm{C}$ A McCarthy, ${ }^{3} \mathrm{P}$ N Hawkins, ${ }^{3} \mathrm{C}$ J Whelan. ${ }^{1}$ National Amyloidosis Centre and UCL Centre for Nephrology, UCL Division of Medicine, Royal Free Hospital, London, UK; ${ }^{2}$ National Amyloidosis Centre, UCL Division of Medicine, Royal Free Hospital, London, UK ${ }^{3}$ National Amyloidosis Centre, UCL Medical School, Royal Free Hospital, London, UK; ${ }^{4}$ National Amyloidosis Centre, London, UK; ${ }^{5}$ The Heart Hospital, UCL Medical School, London, UK; ${ }^{6}$ National Amyloidosis Centre, UCL Medical School, University of London, London, UK; ${ }^{7}$ St George's Hospital, University of London, London, UK

Senile systemic amyloidosis (SSA) is a rare cause of heart failure due to the deposition of wildtype transthyretin. The clinical features and outcome are ill defined; our aim was to evaluate the natural history of the disease in the UK in a group of thoroughly characterised patients. The series included all cases of biopsy proven transthyretin (TTR) amyloidosis with wildtype TTR gene sequencing who were prospectively followed up between January 2001 and May 2010. Clinical, biochemical, ECG and echocardiographic evaluation were performed at presentation to our centre. Patient survival was estimated using Kaplan-Meier analysis. 55 patients with histologically proven SSA; 36 (65.5\%) from cardiac, 14 (25.4\%) from GI tract, 3 (5.5\%) from bladder, 1 (1.8\%) from fat and $1(1.8 \%)$ from carpal tunnel tissue were identified. 49 (89\%) were male. The median age at diagnosis and death were 74 (range 66-89) and 79 (range 69-84) years respectively. Survival from symptom onset and diagnosis was 7.04 (range 0.54-8.41) and 4.58 (range $0.07-5.41$ ) years respectively. In recent years more patients have been diagnosed with 2 (3.6\%), $14(25.5 \%)$ and $39(70.9 \%)$ patients between 2001-2003, 2004-2006 and 2007-2009 respectively. The most common presentation was with breathlessness in 28 patients $(51 \%)$. Twenty-four patients $(43.6 \%)$ had prior carpal tunnel operations. Twelve $(21.8 \%)$ patients had a history of ischaemic heart disease. Fifteen had had a coronary angiogram; 8 were reportedly normal and 7 required intervention. Arrhythmias were common, 20 patients (36.3\%) had a history of atrial fibrillation and $6(10.9)$ had pacemakers in situ. ECG findings were; 24 (43.6\%) in AF, $6(10.9 \%)$ first degree block, 10 (18.2\%) left bundle and $6(10.9 \%)$ right bundle branch block, 27 (49\%) Twave changes, $11(20 \%)<5 \mathrm{~mm}$ complexes in all inferior leads. Echocardiographic findings revealed the median IVSd was 1.7 (range 1.1-2.5) cm, median E/A ratio was 2.7 (range 0.79-5.4), E/E' 15.81 (range 7.5-41.1) and ejection fraction was 45.5 (range 13-83)\%. Blood results showed; the median baseline NT-proBNP was 356.1 (range 5-2611) and troponin T 0.03 (range $0.01-0.28)$. Twenty-five patients had a troponin $\mathrm{T}>0.03(45 \%)$. Ten patients $(18 \%)$ had a detectable paraprotein and $2(3.6 \%)$ had bence jones proteins. SSA is present in $>25 \%$ of the very elderly at post mortem but was rarely diagnosed during life. It is becoming more frequently recognised perhaps due to widespread use of cardiac MRI. Most patients are male but women can be affected. A history of carpal tunnel syndrome is common. The diagnosis is often made after the onset of breathlessness. Systolic and diastolic dysfunction 